\title{
Asymmetric Target Patterns in One-Dimensional Oscillatory Media with Genuine Nonlocal Coupling
}

\author{
F. Plenge, ${ }^{1}$ H. Varela, ${ }^{2}$ and K. Krischer ${ }^{2, *}$ \\ ${ }^{1}$ Fritz-Haber-Institute of the Max-Planck-Society, Faradayweg 4-6, 14195 Berlin, Germany \\ ${ }^{2}$ Physik Department E19, TU München, James-Franck-Str. 1, 85748 Garching, Germany
}

(Received 14 October 2004; published 17 May 2005)

\begin{abstract}
We report the observation of a self-organized asymmetric wave source in a one-dimensional (1D) electrochemical system, namely, the hydrogen oxidation reaction on $\mathrm{Pt}$ in the presence of poisons. Numerical simulations reveal that the nonlocal migration coupling is crucial for the endogenous persistence of the perturbation that causes the asymmetry. Experiments and simulations agree perfectly for the regular and irregular variant of these asymmetric waves, manifesting the existence of nonlocal coupling induced patterns also in physical systems.
\end{abstract}

DOI: 10.1103/PhysRevLett.94.198301

The study of pattern formation in extended nonequilibrium systems has led to a basic understanding of how coherent, spatiotemporal structures form in systems from widely different fields, such as physics, chemistry, or biology. The type of pattern which may arise in a certain medium crucially depends on the way in which different sites of the medium are spatially coupled. So far, the overwhelming part of studies focused on the two limiting cases of spatial coupling, namely, local or nearest neighbor coupling, and global all-to-all coupling with infinite coupling range. Lately, also the dynamics of systems in which both local and global coupling coexist has been investigated (see, e.g., [1-6]).

However, just recently it has been recognized that also the study of intermediate coupling ranges is of central importance: on the one hand, this nonlocal spatial coupling is realized in many natural systems, most notably neural tissues [7]. On the other hand, it was realized that nonlocal coupling may lead to peculiar, novel dynamics, not found in locally or globally coupled systems. Up to date, there are only a few, mainly theoretical, studies that shed some light on the impact of nonlocal coupling on pattern formation [8-13], but we are still far from a broad understanding. Moreover, experimental validations of theoretically predicted new dynamical states are missing.

A class of systems with intrinsic nonlocal coupling are electrochemical systems $[14,15]$. In a previous paper [16], some of us reported novel types of experimentally observed patterns arising during an electrochemical reaction, however, without being able to explain the origin of the unusual dynamics. In this Letter, employing a realistic model, we demonstrate that some of the patterns are indeed unique to systems with nonlocal coupling, thus bridging, to our knowledge for the first time, the gap between experimental observations and theoretical predictions of dynamical states in nonlocally coupled systems.

The decisive variable for the dynamics of electrochemical systems is the voltage drop over the solid-liquid interface, the so-called double layer potential, $\phi_{\mathrm{DL}} \cdot \phi_{\mathrm{DL}}$ is
PACS numbers: 82.40.Np, 05.45.-a, 05.65.+b, 82.45. $-\mathrm{h}$

linked to the electric potential within the electrolyte, $\phi$, by the voltage, which is externally applied between the working electrode (WE) and the counterelectrode (CE). Since the electrolyte is an electroneutral medium, the electric potential distribution in the electrolyte obeys the Laplace equation, $\phi_{\mathrm{DL}}$, being a boundary condition to it. Hence, local perturbations in $\phi_{\mathrm{DL}}$ prompt a redistribution of the electric potential in the entire electrolyte. Consequently, also the normal components of the electric potential at the working electrode change along the entire interface, which, in turn, leads to a position-dependent charging of the double layer, or, equivalently, change in $\phi_{\mathrm{DL}}$ [see below last term of Eq. (1)]. Thus, spatial inhomogeneities in $\phi_{\mathrm{DL}}$ are felt by a whole range of neighboring sites with different strength, which makes the coupling nonlocal. Furthermore, depending on the operation mode and the arrangement of the electrodes, an additional global coupling is present in the system [17-19].

The above mentioned experiments [16] were carried out with the oscillatory hydrogen oxidation reaction (HOR) in the presence of poisons on a Pt ring. The dimensions of the ring were chosen such that pattern formation occurs only along the angular direction. Among the novel patterns a pattern termed asymmetric target pattern (A-TP) was found. Here, two waves originate from a source point and start to travel around the ring, but one of them stops traveling at a fixed location close to the source point and waits there until it is annihilated through a collision with the other one that has been surrounding the ring. This process continued in a stable, oscillatory manner with intervals of constant length in which the potential distribution stayed seemingly homogeneous separating the emission of pulses. Up to now, the origin of this behavior has remained unknown. We show in this Letter that the symmetry breaking of the oscillatory state giving rise to this peculiar pattern originates from the interplay of global and nonlocal coupling.

The mechanism leading to oscillations during the HOR is well understood and involves two species that adsorb on 
the Pt surface and poison the electrode for hydrogen oxidation [20]. An opposite potential dependence of the respective adsorption and separated time scales are crucial for the oscillation mechanism. To obtain a quantitative picture, also the concentration of the inhibiting species in the reaction plane has to be included.

In this Letter spatiotemporal simulations are presented that yield insight into the origin of experimentally observed patterns during the HOR in the presence of poisons and thus clarify the dynamic features necessary to obtain A-TPs. The local dynamics is modeled by a reduced four variable model presented in [20]. The spatial coupling mediated by the electric field is by far more effective than diffusion of the other species. In fact, only the spatial coupling on $\phi_{\mathrm{DL}}$ has to be considered, i.e., the global and the migration coupling [21].

Simulations are carried out in 1D and periodic boundary conditions are imposed. In the following, a dimensionless form of the reduced model equations is used. The transformations of time, space, potential, and concentrations, as well as the definitions of the dimensionless parameters, are given in [22]. The resulting dynamical system is

$$
\begin{gathered}
\dot{\phi}_{\mathrm{DL}}=-i_{r}+\frac{\sigma}{\beta}\left(U-\phi_{\mathrm{DL}}\right)+\frac{\sigma \rho}{\beta}\left(\left\langle\phi_{\mathrm{DL}}\right\rangle-\phi_{\mathrm{DL}}\right) \\
-\left.\frac{\sigma(1+\rho)}{\beta}\left(\frac{\partial \phi}{\partial z}-\phi\right)\right|_{0} \\
\tau_{u} \dot{u}=(1+\chi v)(1-v-u) e^{\phi_{\mathrm{DL}}}-p_{u} u e^{-\phi_{\mathrm{DL}}}, \\
\tau_{v} \dot{v}=k_{v}^{a}-k_{v}^{d} \\
\dot{c}=1-c-\eta \tau_{v}^{-1}\left(k_{v}^{a}-k_{v}^{d}\right),
\end{gathered}
$$

with

$$
\begin{gathered}
k_{v}^{a}-k_{v}^{d}=c(1-v-u) e^{-a \phi_{\mathrm{DL}}}-p_{v} v e^{a \phi_{\mathrm{DL}}}, \\
i_{r}=c_{1}(1-v-u)\left[1-2\left(1+e^{c_{2} \phi_{\mathrm{DL}}}\right)^{-1}\right] .
\end{gathered}
$$

The variables included in the model are the double layer potential, $\phi_{\mathrm{DL}}$, and the coverage of the electrode by the activatory poison $u$ and the inhibitor $v$. Additionally, the concentration of the inhibitory poison in the reaction plane, $c$, is taken into account. $\sigma / \beta$ essentially denotes the dimensionless resistivity of the electrolyte. $\rho$ is the global coupling strength. $\tau_{u / v}$ denote the dimensionless relaxation times of the activatory and inhibitory coverage, respectively. $\chi$ is the only free parameter introduced to model the reported enhanced adsorption rate of $u$ in the presence of $v$ [23]. $\eta$ defines the relative speed of $v$ adsorption/desorption with respect to diffusion from the bulk electrolyte to the reaction plane. $a$ governs the potential dependence of $v$ adsorption/desorption and $p_{u / v}$ determine the adsorption/desorption equilibrium potential of $u$ and $v$, respectively. The reaction current is fitted to theoretically derived data using a more extensive model.
The form of the fit function is given in Eq. (5); the fit parameters $c_{1}$ and $c_{2}$ are given in Table I. It is important to note that the model predictions are obtained using a large number of physical constants which are almost exclusively taken out of the literature and are thus obtained independently and not fitted to the experiment. The meaning and the values of the original parameters are given in [20]. The resulting nondimensional parameters are compiled in Table I. The given values are used throughout this Letter if not mentioned otherwise.

The spatial coupling acting on $\phi_{\mathrm{DL}}$ is composed of a global and a nonlocal coupling [the second last and last term in Eq. (1), respectively]. The nonlocal coupling term describes the difference between the migration currents entering the interface at a position $x$ (which is proportional to the normal derivative of $\phi$ at the interface) and the ones that would flow in a homogeneous situation (which with our nondimensional variables and the boundary condition used is simply proportional to $\phi) . \phi$ and $\phi_{\mathrm{DL}}$ are linked to each other through the potentiostatic control condition $\left(U=\phi_{\mathrm{DL}}+\left\langle\left.\phi\right|_{\mathrm{RE}}\right\rangle\right)$, and at the CE there is an equipotential plane $\left(\left.\phi\right|_{1}=0\right)$. Since Laplace's equation is valid in the entire electrolyte $(\Delta \phi=0), \phi_{\mathrm{DL}}$ is the only time dependent boundary condition of $\phi$, and thus Eq. (1) is a closed expression. Expanding $\phi_{\mathrm{DL}}$ and $\phi$ in a Fourier series, evolution equations for the Fourier components of $\phi_{\mathrm{DL}}$ are obtained assuming a two-dimensional cell geometry [15].

Figure 1 displays the calculated spatiotemporal evolution of $\phi_{\mathrm{DL}}$ for a typical so-called "regular" A-TP. We term the point of the origin of the two waves source point and the point at which one of the waves stops and awaits its annihilation cone point. It has to be stressed that no artificial inhomogeneities have been added in the simulations. The cone point can also behave in the opposite way than presented in Fig. 1. Instead of staying passive after the first wave is stopped, it turns active and waits until the second wave arrives and turns passive again upon collision with it (not shown). The source as well as the cone point travel along the ring with equal and constant speed for all regular A-TPs found. The typical speed of the source point is of the order $10^{-4}$.

The pulse emitted in one direction undergoes shape modulations on its way around the ring resembling aperiodic pulse modulations. Indeed, regular A-TPs coexist with chaotically modulated pulses. On the other hand, regular A-TPs also coexist with target patterns in different regions in parameter space. Because of the presence of a strong

TABLE I. Values of the nondimensional parameters used in the simulations if not stated otherwise

\begin{tabular}{lcc}
\hline \hline$\sigma=0.8$ & $\beta=2.8$ & $\tau_{u}=8.38 \times 10^{-4}$ \\
$\tau_{v}=21$ & $p_{u}=498$ & $p_{v}=3.14 \times 10^{-15}$ \\
$\eta=1.74 \times 10^{4}$ & $c_{1}=4.9$ & $c_{2}=39.6$ \\
$a_{C u}=38 / 3$ & $U=13$ & \\
\hline \hline
\end{tabular}




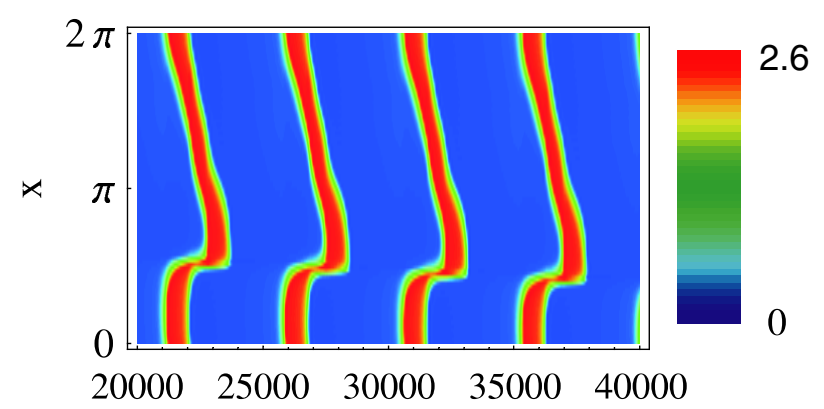

t

FIG. 1 (color online). Space-time plot of $\phi_{\text {DL }}$ of a regular ATP. Red (medium gray) denotes high (passive) and blue (dark gray) low (active) $\phi_{\mathrm{DL}}$ throughout this Letter (cf. color map with maximal and minimal values of $\phi_{\mathrm{DL}}$, respectively).

spatial perturbation in the A-TP motion, A-TPs are favored for initial conditions also exhibiting such an inhomogeneity. Nevertheless, regular A-TPs can also be observed starting from homogeneous initial conditions. A-TPs are generally favored for initial conditions exhibiting a spatial asymmetry and a region in space in which a significant phase mismatch in the individual variables exists. Note also that the so-termed regular A-TPs display apparently aperiodic time series [not visible in Fig. 1 but verified with long time integrations $\left(10^{4}\right.$ periods)].

Apart from regular A-TPs, also irregular A-TPs are found. During irregular A-TP dynamics the source as well as cone points travel in irregular motions along the ring. Additionally, the pulse modulations are strong enough to promote the formation and extinction of a second source point that also leads to a second cone point. The resulting dynamics is displayed in Fig. 2. Short periods of clusterlike behavior interrupt the A-TP motion. After such a clusterlike phase the direction of the pulse traveling around the ring might be interchanged; cf. Figure 2.

The crucial influence of the nonlocal coupling on the formation of the A-TP can be investigated by comparing the above results with the dynamics obtained with a diffusional coupling acting on $\phi_{\mathrm{DL}}$ while the global coupling is kept constant. The diffusion constant is chosen such that the dynamics of the first inhomogeneous mode is equivalent in the nonlocal and diffusional case [i.e., $D=$

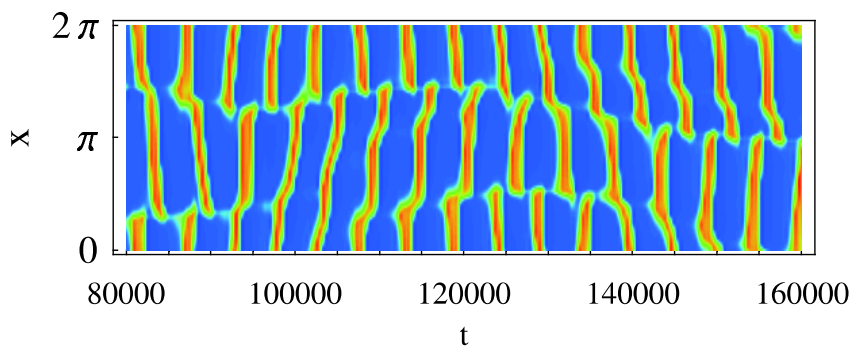

FIG. 2 (color online). Space-time plot of $\phi_{\mathrm{DL}}$ of a irregular ATP. Parameters: $U=4, p_{v}=3.14 \times 10^{-13}$; others as given in Table I. $\sigma(\operatorname{coth}(\beta)-1 / \beta)]$. A-TPs could not be observed using this diffusional coupling. Starting the simulations with an A-TP solution yielded target patterns within one oscillatory period. This behavior can be understood by comparing the contributions of the spatial coupling terms on the righthand side of Eq. (1) for nonlocal and diffusional coupling at the leading front of the cone region, as can be seen in Fig. 3 at an instant in time at which the leading front of the cone point stops moving. Several features hint at the suppression of the A-TP. First, the amplitude of the diffusional coupling is approximately 10 times the one of the nonlocal coupling. However, even if the maximum amplitude of the diffusional coupling is matched to the nonlocal one (thus losing the match in the dynamics of the first mode), A-TPs are not retained. Second, the diffusional coupling function displays a negative region at the cone point, whereas the nonlocal coupling function is positive in the vicinity of the cone point. Hence, even the absolute values of $\phi_{\mathrm{DL}}$ are shifted towards the extinction of the cone point as a result of the nearest neighbor diffusional coupling. Thus, A-TPs can be classified as a true nonlocal coupling phenomenon.

The first experimental observation of an A-TP was reported in [16] and is reprinted in Fig. 4(a) for a convenient comparison with the theoretical data given in Figure 1. The model presented here reproduced the spatiotemporal behavior and the global current for the first time. (See [24] for 3D animations and current time series of the experimental and theoretical data.) Furthermore, these theoretical results suggest that A-TPs and target patterns observed for different applied voltages are no global attractors but coexist, the mixed state reported in [16] resulting from a glueing bifurcation of these two attractors. This hypothesis is supported by recent experiments.

In [16] the question was raised if the asymmetric behavior observed in experiment is due to nonuniformities on the electrode surface. The observation of an irregular A-TP [Fig. 4(b)], which perfectly resembles the simulated pattern presented in Fig. 2, enables us now to negate this question. In the irregular A-TP the cone point occurs at

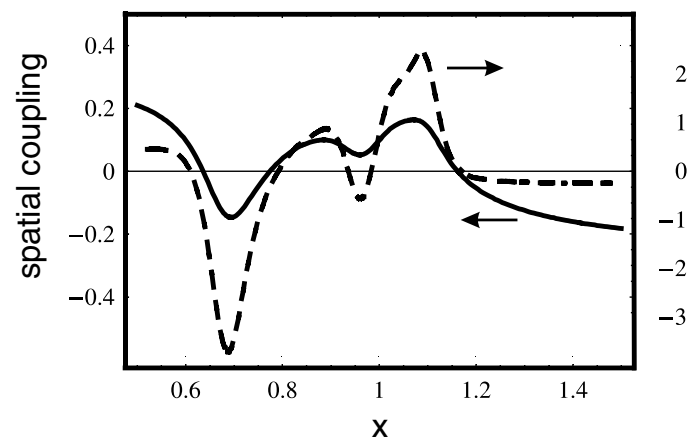

FIG. 3. Comparison of the total spatial coupling terms at the leading front of the cone region of the regular A-TP at $t=5100$, i.e., at an instant in time at which the leading front of the cone point stops moving; solid line: nonlocal and global coupling; dashed line: diffusional and global coupling. 


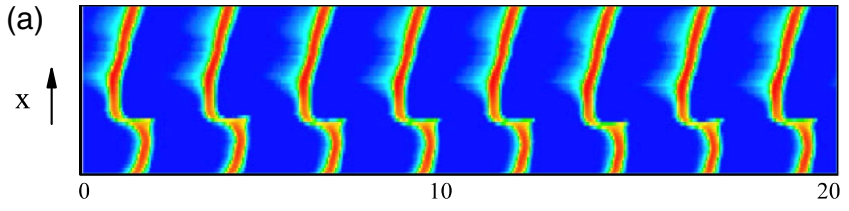

$\mathrm{t}[\mathrm{s}]$

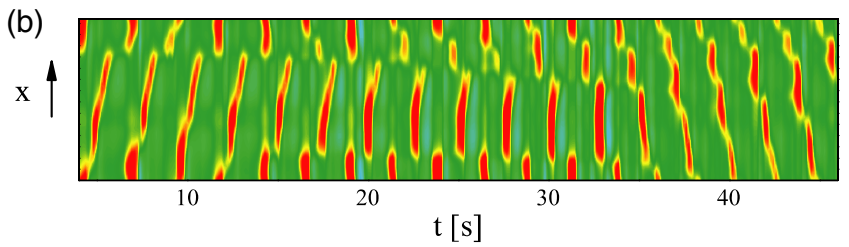

FIG. 4 (color online). Experimental manifestations of (a) regular A-TP and (b) irregular A-TP. In (b) the difference of the signal corresponding to the double layer potential from its mean value at every time step is plotted. For experimental details see [16,27], respectively.

random positions along the entire ring; surface nonuniformities, in contrast, would cause its preferred occurrence at particular positions.

On the first sight similar but structurally different phenomena where a pulse is emitted from a source in just one direction in 1D media were reported by Perraud et al. (1D spirals) [25] and Stich [26]. The first one was attributed to the interaction between Turing and Hopf modes; the latter involves interaction between self-organized pacemakers and pulses.

The theoretical results reproduce the novel experimentally observed patterns with outstanding consilience. Thus, it was shown not only that the homogeneous dynamics is captured by the model Eqs. (1)-(4), but also that the reduced model is sufficient to describe the previously uncaptured peculiar A-TP dynamics. The only spatial coupling mechanisms taken into account are the nonlocal coupling and the global coupling acting on $\phi_{\mathrm{DL}}$, A-TPs being a genuine nonlocal coupling pattern observed both in experiment and theory. It should be noted that the global coupling strength used in the simulations exceeded those in the experiments.

To gain further insight into the interaction of the local dynamics and the nonlocal and global spatial couplings, the introduction of a "toy model" that preserves the phenomenological spatiotemporal behavior, but is reduced to the minimal number of nonlinearities and variables necessary to capture the described behavior, would be desirable. Furthermore, it can be expected that many more examples of unusual patterns that arise due to a nonlocal interaction between different sites in nonlinear media are to be discovered.

Financial support of the DFG in the framework of the SFB 555 is gratefully acknowledged.
*Electronic address: krischer@ph.tum.de URL: http://www1.physik.tu-muenchen.de/lehrstuehle/ e19/krischer.html

[1] D. Battogtokh and A.S. Mikhailov, Physica D (Amsterdam) 90, 84 (1996).

[2] E. Schöll, Nonlinear Spatio-Temporal Dynamics and Chaos in Semiconductors, Cambridge Nonlinear Science Series Vol. 10 (Cambridge University Press, Cambridge, England, 2001).

[3] D. Luss and B. Warwaha, Chaos 12, 172 (2002).

[4] V. K. Vanag et al., Nature (London) 406, 389 (2000).

[5] M. Kim et al., Science 292, 1357 (2001).

[6] V. Zykov et al., Phys. Rev. Lett. 92, 018304 (2004).

[7] B. Ermentrout, Rep. Prog. Phys. 61, 353 (1998).

[8] Y. Kuramoto and H. Nakao, Phys. Rev. Lett. 76, 4352 (1996).

[9] Y. Kuramoto, D. Battogtokh, and H. Nakao, Phys. Rev. Lett. 81, 3543 (1998).

[10] D. Battogtokh and Y. Kuramoto, Phys. Rev. E 61, 3227 (2000).

[11] E. M. Nicola et al., Phys. Rev. E 65, 055101(R) (2002).

[12] D. Tanaka and Y. Kuramoto, Phys. Rev. E 68, 026219 (2003).

[13] D. M. Abrams and St. H. Strogatz, Phys. Rev. Lett. 93, 174102 (2004).

[14] N. Mazouz, G. Flätgen, and K. Krischer, Phys. Rev. E 55, 2260 (1997).

[15] K. Krischer, in Advances in Electrochemical Science and Engineering, edited by R.C. Alkire and D. M. Kolb (Wiley-VCH, Weinheim, 2003), p. 89.

[16] P. Grauel, H. Varela, and K. Krischer, Faraday Discuss. 120, 165 (2002).

[17] J. Christoph et al., J. Chem. Phys. 110, 8614 (1999).

[18] P. Grauel et al., J. Phys. Chem. B 102, 10264 (1998).

[19] N. Mazouz, G. Flätgen, K. Krischer, and I. G. Kevrekidis, J. Electrochem. Soc. 145, 2404 (1998).

[20] F. Plenge et al., Z. Phys. Chem. (Frankfort/Main) 217, 365 (2003).

[21] It was tested if the diffusion of the other activatory variable would introduce new effects, which was not the case.

[22] F. Plenge, Ph.D. thesis, TU Berlin, 2003, http://edocs.tuberlin.de/diss/2003/plenge/florian.htm.

[23] V. Stamenković and N. M. Marković, Langmuir 17, 2388 (2001).

[24] See EPAPS Document No. E-PRLTAO-94-065520 for 3D animations and current time series of the experimental and theoretical data. A direct link to this document may be found in the online article's HTML reference section. The document may also be reached via the EPAPS hompage (http://www.aip.org/pubservs/epaps.html) or from ftp.aip.org in the directory /epaps/. See the EPAPS homepage for more information.

[25] J.-J. Perraud et al., Phys. Rev. Lett. 71, 1272 (1993).

[26] M. Stich, Ph.D. thesis, TU Berlin, 2003.

[27] H. Varela, Ph.D. thesis, FU Berlin, 2003, http://darwin. inf.fu-berlin.de/2003/304/. 